\title{
«Observar TV»:
}

Un observatorio infantil de televisión para la interlocución de los niños

\author{
A Children's Observatory of Television: «Observar TV», a Space \\ for Dialogue between Children
}

\section{RESUMEN}

Este artículo presenta los resultados de "Observar TV», un observatorio infantil de televisión puesto en marcha en la ciudad de Barranquilla (Colombia) en el que los niños y niñas participantes actuaron como protagonistas de los procesos de investigación, formación e interlocución llevados a cabo. El proyecto apuntó a: 1) La reflexión y discusión de las preferencias y opiniones que niños/as presentaron en relación con la televisión; 2) La identificación e interpelación de sus imaginarios de la ciudadanía alrededor de la convivencia y la paz, la interculturalidad y la participaciónresponsabilidad democrática; 3) El desarrollo de procesos de formación que contribuyeran a cualificar su capacidad crítica en torno a la televisión y su consumo; 4) La visibilización de los niños/as como interlocutores en la agenda pública de su ciudad. El proceso se desarrolló desde la investigación-acción participativa utilizando técnicas cualitativas (grupos focales, talleres y observación), para la ejecución de tres componentes simultáneos: investigación, formación y visibilización/interacción. Los resultados permitieron constatar que los chicos se presentaron críticos hacia la televisión, dando a conocer sus gustos y disgustos en relación con los contenidos. También que ellos asumieron el reto de discutir, transformar o reafirmar sus imaginarios de ciudadanía, y fortalecieron su capacidad expresiva al vincular las reflexiones surgidas en el observatorio dentro del programa «Aprender a ver TV», del canal regional «Telecaribe».

\section{ABSTRACT}

This paper presents the results of work on «Observar TV», a children's observatory of television launched in the city of Barranquilla (Colombia) in which children participated as protagonists in research, training and spaces for dialogue centred on their observations of television broadcasts. The goals of the project included: 1) to ascertain the reflection and discussion of preferences and opinions (expressed by boys and girls) regarding television; 2) to identify and discuss their imagery around citizenship in relation to coexistence and peace, multiculturalism and democratic responsibility and participation; 3 ) to understand the development processes that contribute to the construction of their critical thinking abilities regarding television and its consumption; 4) to increase the visibility of children as active participants in setting the public agenda of their city. This study was developed using a participatory action research approach that employed qualitative methods (focus groups, workshops, and observations) for the simultaneous execution of three components: research, training, and visibility/interaction. The results demonstrate that the child participants displayed a critical attitude toward television and expressed their likes and dislikes regarding television content. The participants took up the challenge to discuss, transform, or reaffirm their imagery relating to citizenship and strengthened their capacities in language and communication. The children expressed the reflections that came up in the work of the observatory by participating in the television program «Learning to watch television», that was produced by the regional station «Telecaribe».

\section{PALABRAS CLAVE / KEYWORDS}

Niños, televisión, educación en medios, interactividad, capacidad crítica, imaginarios de ciudadanía, escuela.

Children, television, media literacy, interactivity, critical capacity, imagery of citizenship, school.

* Jair Vega-Casanova es Profesor Investigador del Departamento de Comunicación Social de la Universidad del Norte en Barranquilla (Colombia) (jvega@uninorte.edu.co).

* Andrea Lafaurie-Molina es Profesora Investigadora del Instituto de Estudios en Educación de la Universidad del Norte en Barranquilla (Colombia) (andrelafour@gmail.com). 


\section{Introducción}

Desde hace varios años, alrededor de las investigaciones que exploran la relación televisión y niños/niñas se ha reconocido la presencia de una tendencia centrada en el análisis de los efectos de una televisión nociva sobre unas audiencias consideradas pasivas y fácilmente influenciables, la cual incluso se mantiene en algunos estudios (Rodríguez, 2005).

En otra perspectiva, algunos investigadores han apostado por la capacidad activa y crítica de niños y niñas, considerándolos ciudadanos capaces de interpretar y resignificar los mensajes de los medios (Ferrés, 1994; López de la Roche \& al., 1998; Aguaded, 1999; 2005). Charles y Orozco (1990) sostienen que el niño televidente no nace, se hace, razón por la que es necesario generar en él una actitud crítica, reflexiva y creativa que sustente su papel dinámico en la comunicación a través de procesos de mediación.

Un tercer enfoque, que aparece coincidente con el anterior, considerando las audiencias como ciudadanos activos, añade como sentido prioritario el fortalecimiento de la visibilización de esta capacidad crítica para generar acciones en la esfera pública (Alfaro y Quezada, 2006). Sin embargo, tal como sostiene Rincón (2008) son pocas las investigaciones de audiencias que afectan la producción de los medios, es decir, que transcienden la opción de conocer a las audiencias/consumidoras para promover su tránsito hacia audiencias/productoras.

Situándonos concretamente en la relación niños/as, televisión y ciudadanía, los resultados de algunas investigaciones muestran que los menores suelen hacer uso de un discurso moralista, frecuentemente adoptado de la familia o la escuela, para referirse a la ciudadanía o para valorar la bondad o maldad de los contenidos televisivos (Vega \& Castro, 2006).

A partir de lo anterior, se ha identificado la necesidad de proveer los espacios necesarios para la expresión, interpelación e interlocución de imaginarios de ciudadanía de los infantes, vinculando en el proceso a las instituciones que influyen en la configuración de los mismos. En ese sentido, se propuso el diseño de «Observar TV", un observatorio infantil de televisión en la ciudad de Barranquilla (Colombia), sustentado en que los niños/as ejercieran como protagonistas de los procesos de investigación y formación, diferenciándose de los observatorios de televisión infantiles, en los cuales son los adultos quienes discuten y critican las programaciones y contenidos.

Los objetivos perseguidos con la puesta en marcha de «Observar TV» fueron: 1) Identificar las preferencias y opiniones de los niños y niñas en relación con la oferta televisiva; 2) Conocer los imaginarios de ciudadanía que ellos y ellas identifican con respecto a la convivencia y paz, la interculturalidad y la participación-responsabilidad democrática; 3) Desarrollar actividades para la interpelación de estos imaginarios de ciudadanía; 4) Desarrollar procesos de formación que contribuyeran a cualificar su capacidad crítica alrededor de la televisión y su consumo; 5) Generar espacios y redes para propiciar la interlocución/interactividad de los niños/as con la televisión y su visibilización como ciudadanos en la agenda pública de su ciudad.

\subsection{Interpelación de imaginarios sobre ciudadanía}

La revisión de la literatura permite constatar que no existen muchas referencias en torno a la investigación de los imaginarios de ciudadanía, y menos aún que prioricen el estudio de sus interpelaciones o hagan énfasis en la población infantil. Los pocos estudios encontrados plantean que estos desempeñan un papel clave en la convivencia y la ciudadanía, en tanto las imágenes colectivas que se construyen sobre lo que es ser un ciudadano constituyen un referente para sus acciones, destacando también el papel que juegan en la configuración de estos imaginarios los medios de comunicación, dentro de ellos la televisión (Vega \& García, 2008). Esta construcción parte del concepto de imaginarios de Pintos (2000), entendido como «esquemas, construidos socialmente, que nos permiten percibir algo como real, explicarlo e intervenir operativamente en lo que en cada sistema social se considere como realidad», así como de una discusión sobre el sentido de la ciudadanía presentada por Pineda (1999), en relación con el rol que juegan los individuos y las comunidades frente al poder, el Estado y el desarrollo. En el enfoque del súbdito o beneficiario, clásicamente introducido por Hobbes, se identifica al ciudadano como súbdito que asume acríticamente las determinaciones del Estado/gobernantes, entendiendo que en la medida en que se someta al poder, sin obstruir sus decisiones, recibirá sus beneficios. En el liberal, cuyo principal exponente es John Locke, se reconoce la ciudadanía como participación de los ciudadanos en igualdad de condiciones en el marco de las leyes, las cuales le otorgan derechos y deberes. Este participa en las decisiones del gobierno, mientras los gobernantes están sujetos al control, juicio y aprobación de los ciudadanos. El enfoque del empoderamiento, clásicamente defendido por Rousseau, critica la pretendida igualdad de los ciudadanos ante la ley y en las condiciones de participación. Por tanto, propone la necesidad de convertir a los individuos en sujetos de poder (Mouffe, 1999). 
Ahora bien, en relación con la interpelación de imaginarios, Buenfil (Vega \& Mendivil, 2012: 447) sostiene que en la práctica de la interpelación el agente se sitúa como sujeto activo, capaz de incorporar nuevos elementos valorativos, conductuales o conceptuales para transformar radicalmente su práctica cotidiana o hacer una reafirmación más fundamentada. En un trabajo específico con jóvenes, estos mismos autores propusieron la interpelación de imaginarios como «la discusión sobre los supuestos, en el planteamiento de la pregunta o la afirmación que permite hacer visible lo que está oculto detrás de lo concebido como verdad», promoviendo el cuestionamiento de los imaginarios y sus implicaciones a través de procesos dialógicos colectivos.

En «Observar TV» se consideraron los imaginarios de ciudadanía en relación con las competencias ciudadanas definidas por el Ministerio de Educación Nacional de Colombia (2004): convivencia y paz, interculturalidad y participaciónresponsabilidad democrática. Las competencias en convivencia y paz plantearon la necesidad de valorar el conflicto como parte de la convivencia y aceptación del otro, el ejercicio de libertad de elección y la responsabilidad individual y colectiva. Las competencias en interculturalidad se sustentaron en el entendimiento de sociedades plurales en las que las diferencias puedan ser celebradas positivamente como elemento de afirmación de las identidades (Mouffe, 1999). Las competencias dirigidas a la participaciónresponsabilidad democrática fueron relacionadas con el modelo de construcción de ciudadanía basado en el empoderamiento que considera la creación y fortalecimiento de escenarios para el debate.

\subsection{Educación en medios para niños y niñas}

Alrededor de la educación en medios se reconocen discusiones que datan de la década de los sesenta, momento desde el cual ha prevalecido una orientación proteccionista ligada a la necesidad de contrarrestar las actitudes o conductas indeseables promovidas por los medios, aduciendo su falta de valor cultural en relación con el arte o la literatura clásica. Se consideraba que los medios ejercían influencias negativas sobre niños, niñas y jóvenes, quienes se presentaban vulne- rables a su manipulación, mientras que los profesores se consideraban fuera del alcance mediático siendo capaces de identificar sus intenciones y en ese sentido, ofrecer herramientas de liberación y protección a sus estudiantes.

Las perspectivas más recientes han advertido la limitación de tales concepciones y han apostado por situar a los medios como instancias culturales complejas y relevantes para unas audiencias con autonomía, crítica, capacidad de decisión y participación (Buckingham, 2005), concibiéndose así la educación en medios como un proceso que implica la comprensión crí- 
en medios en los que estos sean entendidos como espacios de expresión cultural.

\subsection{Audiencias infantiles e interactividad}

Desde que a comienzos del presente siglo se señalara a niños, niñas y jóvenes como nativos digitales (Prensky, 2001), varias cosas se han asumido alrededor del uso y relaciones que este tipo de audiencias establecen con los medios y tecnologías, entre ellas, una

cepción sobre la capacitación para la expresión (Orozco $\&$ al., 2012).

Koolstra \& Bos (2009) señalan que la investigación alrededor de la interactividad, popularizada desde los años ochenta, ha estado caracterizada por la identificación de propiedades interactivas en medios y procesos de comunicación, así como por el estudio de las percepciones que tienen las audiencias y los productores en relación con la interactividad, prevaleciendo una perspectiva tecnológica sobre el análisis de los procesos de comunicación interactiva y sus implicaciones. Referente a la investigación con niños, niñas y jóvenes, la interactividad es principalmente concebida como componente de la comunicación para impulsar el logro de objetivos concretos (Masterman \& Rogers, 2002; Chang \& al., 2010; Dezuanni \& MonroyHernández, 2012), o como categoría particular dentro del consumo genérico de medios, observando el desarrollo de acciones concretas: envío de emails, realización de votaciones, hacer intercambios, crear contenidos, etc. (Livingstone $\mathcal{E}$ al., 2005; Aikat, 2005).

En ese sentido, pensar en las posibilidades de interactividad implica diferenciar el con-

mayor disposición a interactuar y ser activos ante las opciones de participación presentes en la oferta mediática y tecnológica.

Sin embargo, algunos autores han advertido que frente a tal optimismo generalizado valdría la pena considerar que el acceso instrumental a la tecnología dista de ser el deseable para todos los sectores sociales, y aún más importante que eso, que situarse como audiencia activa y propositiva dentro de la cultura digital no es aún una situación generalizada. Ello encuentra diversas explicaciones entre las que se señalan: 1) que el cambio cultural necesita de tiempo para efectuarse; 2) que las audiencias están acostumbradas a ser tímidas y no expresar posturas como consecuencia del privilegio que ha ostentado la comunicación masiva vertical durante tantas décadas; 3) que desde el sistema educativo se ha enfatizado el logro de competencias en lectura más que en escritura, lo que puede traducirse en la supremacía de la capacitación para la re- sumo amplificado, descentrado y diferido que promueven los medios y tecnologías digitales de hoy (descargar materiales, elegir cuándo, cómo y dónde visionar contenidos, reaccionar, chatear, jugar), de los procesos productivos, innovadores y trascendentes a través de los cuales los receptores pueden realmente situarse como emisores/productores, consolidando un modo distinto de interlocución, fundamentado en la reflexión y discusión (Orozco \& al., 2012).

En este último contexto, tiene cabida el concepto de agencia humana introducido por Guiddens y citado por Muñoz y Muñoz (2008), referido a que todos los individuos tienen el potencial de autogestionar su propia existencia, aportando a la socialización y significación del mundo de la vida desde su propia creatividad y producción cultural. Así, la agencia humana se sitúa como eje fundamental en la configuración de la ciudadanía cultural de diferentes grupos, entre ellos niños, niñas y jóvenes, cuya presencia resulta altamente signi- 
ficativa dentro de instancias culturales como son los medios de comunicación.

\section{Material y métodos}

"Observar TV»" fue desarrollado en el año 2008

en cinco instituciones educativas públicas y privadas de los estratos socioeconómicos alto, medio y bajo de la ciudad de Barranquilla, con 80 niños y niñas ${ }^{2}$ de los grados $4^{\circ}$ y $5^{\circ}$ de primaria que estaban entre los 7 y 11 años, y cinco profesores, uno en cada institución educativa. Mediante la investigación-acción participativa, utilizando técnicas cualitativas, se ejecutaron tres componentes simultáneos: investigación, formación y visibilización.

El componente de investigación implicó, por un lado, la indagación a través de grupos focales de las opiniones de niños y niñas sobre la televisión, específicamente en relación con su calidad y preferencias de consumo. Para establecer la interpelación de imaginarios de ciudadanía de los niños y niñas, en relación con convivencia y paz, interculturalidad y participaciónresponsabilidad democrática, se estableció una línea de base al comienzo del proceso y una evaluación final ocho meses después, basadas en la utilización de una técnica de reconstrucción de memoria denominada "colcha de retazos». Esta técnica consiste en la elaboración de dibujos por parte de los participantes, a partir de una pregunta que indaga sobre una situación particular vivida o por una imagen configurada desde la experiencia. Según Riaño (2000), permite trascender las opiniones debido a que posibilita construir situaciones específicas, así como la interpretación que de ellas hacen individual y colectivamente los participantes. Los dos procesos se apoyaron también en observación participante.

El componente de formación se desarrolló a partir del análisis de lo investigado alrededor de las opiniones y preferencias del consumo televisivo y de los imaginarios de ciudadanía, utilizando talleres sobre educación en medios (lectura crítica, regulaciones, guiones, producción y postproducción) en los que se enfatizó el desarrollo de procesos de reflexión, discusión e interpelación de imaginarios (ciudadanía, violencia, conflicto, poder y autoridad, género, centro/periferia, orientaciones sexuales), que permitieran a los niños y niñas cimentar posiciones fundamentadas a partir de las cuales ejercer interacciones mediáticas desde su ciudadanía infantil. Ellos y ellas tuvieron la oportunidad de crear guiones de historias audiovisuales propias relacionadas con cada grupo de competencias.

El componente de visibilización/interacción permitió la socialización de los resultados del proceso, si- tuando a los niños y niñas como interlocutores válidos en la agenda pública de la ciudad, cuyas opiniones fueron incluidas en «Aprender a ver TV ${ }^{\text {», un progra- }}$ ma constituido como el espacio de los televidentes en el canal regional de televisión Telecaribe ${ }^{3}$. Se realizaron cinco vídeos en los que los niños y niñas dieron cuenta de las reflexiones y discusiones que se propiciaban en el Observatorio4.

\section{Resultados}

A continuación se presentan los principales resultados. En primera instancia, las opiniones y preferencias que frente a la televisión tuvieron los niños y niñas, posteriormente, se describen tanto los imaginarios iniciales como las interpelaciones de los mismos, en relación con las competencias ciudadanas, convivencia y paz, interculturalidad y participación-responsabilidad democrática.

\subsection{Opiniones y preferencias}

Con respecto a las opiniones y preferencias, niños y niñas reclamaron una televisión de calidad que sea caracterizada por: a) una oferta incluyente en la que se exploren más formatos para la audiencia infantil pues consideraron que hay escasez y saturación de dibujos animados y series importadas; b) la construcción adecuada de personajes, ya que les aburren los que son buenos o malos de principio a fin en las historias y prefieren aquellos más parecidos a sí mismos, con problemas y equivocaciones; c) combinación entre contenido y forma, o sea, que se combine lo educativo con el entretenimiento porque reconocen la existencia de programas que aunque presentan buenos contenidos (enseñan cosas), son muy aburridos; d) la adecuación de horarios, dado que sienten que en las programaciones no tienen en cuenta sus rutinas escolares, de descanso y de actividades complementarias, emitiendo programas en momentos que ellos no los pueden ver.

En cuanto a las regulaciones para ver televisión, niños y niñas comentaron que sus padres y madres les prohíben ver ciertos programas considerados violentos 5 . Sin embargo, alrededor de estas prohibiciones manifestaron que les agradaría más que ellos los acompañaran a ver esos programas y les explicaran las razones de por qué no está bien que los vean, pues piensan que se los prohíben sin conocer de qué van tales contenidos. También desean que exista más diálogo en el momento de ver televisión y negociar la tenencia del control en los espacios familiares.

Asimismo, señalaron que sus padres y madres les generan sentimientos de culpa ya que frecuentemente les mencionan que el ver televisión afecta su rendi- 
miento académico, proyectándose así la educación y el entretenimiento como dos componentes antagónicos.

\subsection{Imaginarios de ciudadanía en relación con la televisión}

Alrededor de los imaginarios de ciudadanía, la mención de «un antes» corresponde a la línea de base levantada al inicio del Observatorio, y el término «resignificación» aduce a la información obtenida tras los procesos de interpelación y educación en medios.

\subsubsection{Imaginarios de convivencia y paz}

En «un antes», los niños y niñas definieron a las personas violentas con estereotipos de personajes que ven en programas o informativos, como «rateros», "gamines», «guerrilleros» $y$ «paramilitares» 6 . Relacionaron los espacios violentos con imágenes de peligro que ven en la televisión, lugares oscuros, solitarios, que huelen feo, habitados por la gente pobre, donde se producen peleas, robos, asesinatos y hay sangre. También vincularon la violencia con la presencia de armas o agresiones físicas, clasificando ciertos programas como violentos, los cuales dicen no ver, adoptando las regulaciones de sus profesores o familiares. Y asumieron el conflicto como una situación negativa que implica peleas y actos violentos, los cuales deben ser resueltos, eliminando las posiciones contrarias a través de acuerdos en los que intervengan autoridades reconocidas como la policía o los políticos.

Las actividades llevadas a cabo para la re-significación de estos imaginarios permitieron observar que niños y niñas criticaron que los noticieros prioricen la violencia en relación con el conflicto armado, pero no que se refieran a otros tipos de violencia como la verbal, que está presente en diferentes contextos de la

vida cotidiana. También comenzaron a valorar el conflicto como expresión de posiciones contrarias, sin que necesariamente se requiera su resolución a través de acuerdos. Y apreciaron la representación de conflictos entre personajes de los programas televisivos, ya que estos ofrecen la oportunidad de aprender acerca de negociación o aceptación de desacuerdos.

\subsubsection{Imaginarios de interculturalidad}

En «un antes», los niños y niñas mencionaron que la televisión suele presentar a los «cachacos» como trabajadores e inteligentes, frente a los «costeños» como personas alegres, irresponsables y «corronchas»?. También que Colombia es representado como un país peligroso, exportador de drogas e inferior a países como Estados Unidos.

En relación con los géneros, dijeron que en la televisión las mujeres son presentadas como débiles, vanidosas y amas de casa, frentes a hombres trabajadores que normalmente se encuentran ocupando altos cargos. Referente a las orientaciones sexuales expresaron que la televisión suele mostrar a los gays ridículamente, siempre en ocupaciones relativas a la belleza, como estilistas o managers de reinas, mientras representan a las lesbianas como «machorras», es decir, mujeres que quieren ser hombres.

La re-significación de imaginarios en torno a la interculturalidad permitió observar posiciones críticas fundamentadas, como por ejemplo, el que las diferencias raciales que presenta la televisión aumentan las fronteras entre blancos y negros, y que los géneros y orientaciones sexuales son fuertemente estereotipados a través de personajes que no necesariamente representan cada identidad. Algunos guiones propuestos por niños y niñas dejaron ver un interés por historias

en las cuáles los perso-

\section{Re-significación de imaginarios}

Antes. Asociación de

\begin{tabular}{|c|c|}
\hline $\begin{array}{l}\text { - La violencia con personajes considerados como } \\
\text { malos en términos del estereotipo bondad y maldad. }\end{array}$ & $\begin{array}{l}\text { - Mirada crítica de los personajes que aparecen } \\
\text { estereotipadamente como buenos o malos. } \\
\text { - Construcción no estereotipada de los mismos en } \\
\text { sus propios relatos. }\end{array}$ \\
\hline $\begin{array}{l}\text { - La violencia con la presencia de armas y agresión } \\
\text { fisica. } \\
\text { - Espacios violentos con lugares peligrosos, oscuros, } \\
\text { solitarios, que huelen feo, habitados por gente pobre, } \\
\text { donde suceden peleas, atracos y asesinatos. } \\
\text { - Programas violentos porque muestran agresiones } \\
\text { físicas, se consume droga o se usan armas. }\end{array}$ & $\begin{array}{l}\text { - Reconocimiento de otros tipos de violencia como } \\
\text { la verbal, más allá de la violencia fisica } \\
\text { exclusivamente relacionada con el conflicto } \\
\text { armado. } \\
\text { - Reconocimiento de que la violencia aparece de } \\
\text { múltiples formas en otros espacios tales como la } \\
\text { familia, la escuela, el trabajo, etc. }\end{array}$ \\
\hline $\begin{array}{l}\text { - El conflicto con situaciones negativas (peleas y } \\
\text { actos violentos) y como resultado de posiciones } \\
\text { contrarias. }\end{array}$ & $\begin{array}{l}\text { - Valoración del conflicto como algo inherente a la } \\
\text { diferencia, que no es ni positivo ni negativo en sí } \\
\text { mismo. } \\
\text { - Valoración de la presencia de conflictos en los } \\
\text { programas de televisión porque de ellos se puede } \\
\text { aprender a negociar y analizar situaciones de la } \\
\text { vida real. }\end{array}$ \\
\hline $\begin{array}{l}\text { - La resolución c } \\
\text { acuerdos en los } \\
\text { o políticos). }\end{array}$ & $\begin{array}{l}\text { - Aceptación de que el conflicto no necesariamente } \\
\text { lleva a acuerdos sino que pueden prevalecer } \\
\text { posiciones contrarias. }\end{array}$ \\
\hline
\end{tabular}

Tabla 1. Imaginarios de convivencia y paz. najes disten de los estereotipos actuales y luchen por ser reconocidos a partir de sus diferencias. Por ejemplo, unas niñas propusieron presentadoras de televisión gordas.

\subsubsection{Imaginarios de participación, responsabilidad democrática}

Los niños y niñas en «un antes» consideraron como buenos 
ciudadanos a personajes que representaban valores morales como la solidaridad, el respeto, la obediencia, la responsabilidad, la amabilidad, la tolerancia y el cuidado del medio ambiente. $Y$ valoraron como malos ciudadanos a aquellos personajes que se presentaban antagónicos a los buenos, es decir, groseros, rebeldes, agresivos, violentos, irresponsables o ambiciosos. La participación ciudadana fue concebida como la posibilidad de votar en los procesos electorales o asistir a marchas.

En cuanto a la re-significación de imaginarios, se pudo apreciar que niños y niñas comenzaron a valorar otras cualidades alrededor de la ciudadanía, tales como el empoderamiento, la crítica y la capacidad para argumentar, cuestionando también a los personajes que representan la autoridad, pues aunque en algunos programas se suele mostrar a los policías como héroes, a veces estos actúan mal con los ciudadanos. También comenzaron a considerar que estos últimos pueden emprender acciones de acuerdo a sus intereses y convicciones.

\section{Discusión}

Los resultados de «Observar TV» evidenciaron niños y niñas críticos ante la oferta de contenidos, dispuestos a expresar sus gustos, disgustos, opiniones y preferencias en torno a la televisión. Sin embargo, la mediación que alrededor de la recepción están ejerciendo familias y escuelas puede ser tildada de ausente, o en otros casos, limitada y contraproducente al basarse en prohibiciones, amenazas y castigos, ciñéndose a una mediación restrictiva, frente a la cual los niños y niñas dicen echar de menos el que sus padres y madres los acompañen a ver televisión (mediación acompañada) o dialogar con ellos acerca de los contenidos que ven o no deben ver (mediación activa) (Potter, 1998).

Los espacios de investigación constituyeron un proceso de especial relevancia en tanto permitieron que niños $\mathrm{y}$ niñas tomaran con- ciencia de sus imaginarios de convivencia y paz, interculturalidad y participación-responsabilidad democrática. Además, los procesos de interpelación de los mismos permitieron la generación de reflexiones a partir de las cuales fundamentaron o transformaron sus opiniones, lo cual constituyó, junto con sus preferencias en relación con la televisión, la materia prima para interactuar como productores y protagonistas de contenidos emitidos en el canal regional Telecaribe.

Dentro de las posiciones defendidas en los vídeos, niños y niñas manifestaron entre otras cosas, sus gustos y disgustos en relación con la televisión, cómo debe ser una televisión de calidad y la necesidad de valorar el conflicto como parte de la convivencia, desestimando el acuerdo como única salida (Mouffe, 1999). También se mostraron críticos ante las representaciones estereotipadas que proponen los medios alrededor de temáticas como centro-periferia, hombre-mujer y orientaciones sexuales; y señalaron la crítica y la argumentación como características imprescindibles de la participación, sugiriendo una nueva mirada de la ciudadanía coincidente con la perspectiva del empoderamiento (Pineda, 1999).

En ese sentido, niños y niñas de "Observar TV" pudieron enfrentarse al reto de interactuar con la televisión haciendo visible su capacidad crítica como audiencias activas y su capacidad expresiva como productores de contenidos en contextos que posibilitan una interlocución horizontal (Orozco \& al., 2012), valorando especialmente los espacios y procesos que les proporcionan reflexiones y herramientas para desarro-

\begin{tabular}{|c|c|}
\hline Antes & Re-significación de imaginarios \\
\hline $\begin{array}{l}\text { En el ámbito de la televisión nacional, } \\
\text { asociación del centro con estereotipos } \\
\text { mayoritariamente positivos y de la } \\
\text { periferia con estereotipos negativos. }\end{array}$ & $\begin{array}{l}\text { - Se reforzaron argumentos en relación con que la } \\
\text { representación de personajes en la televisión es injusta y } \\
\text { estereotipada ya que los habitantes del centro del país no son } \\
\text { necesariamente trabajadores e inteligentes y los de la costa no } \\
\text { son sólo irresponsables y corronchos. }\end{array}$ \\
\hline $\begin{array}{l}\text { A nivel internacional, asociación de } \\
\text { Colombia con estereotipos negativos } \\
\text { (país peligroso y exportador de } \\
\text { drogas), y Estados Unidos con } \\
\text { estereotipos positivos (pais de mejor } \\
\text { valia). }\end{array}$ & $\begin{array}{l}\text { - Se reforzaron críticas en torno a los estereotipos presentados } \\
\text { en las peliculas de Hollywood y colombianas con respecto a } \\
\text { Estados Unidos y Colombia. } \\
\text { - Se afirmó el deseo de encontrar en la oferta nacional historias } \\
\text { más cercanas a su propio contexto y no mayoritariamente } \\
\text { referentes a la sociedad estadounidense. }\end{array}$ \\
\hline $\begin{array}{l}\text { Identificación de las mujeres como } \\
\text { débiles, vanidosas y amas de casa; } \\
\text { mientras a los hombres como } \\
\text { trabajadores que ocupan altos cargos. }\end{array}$ & $\begin{array}{l}\text { - Desacuerdo acerca de cómo son representadas las mujeres en } \\
\text { la televisión: superficiales, interesadas, sumisas y obedientes. } \\
\text { - Manifestación de que prefieren las telenovelas en las que se } \\
\text { reevalúan esos roles de las mujeres, como «Betty, la fea». } \\
\text { - Cuestionamiento acerca de por qué los hombres no son } \\
\text { representados también como débiles o no trabajadores, tal como } \\
\text { puede suceder en la vida cotidiana. }\end{array}$ \\
\hline $\begin{array}{l}\text { Identificación de estereotipos de los } \\
\text { gays como ridiculos o amanerados. }\end{array}$ & $\begin{array}{l}\text { - Resignificación de los gays como hombres que les gustan otros } \\
\text { hombres, criticando los programas de televisión donde los } \\
\text { ridiculizan. }\end{array}$ \\
\hline $\begin{array}{l}\text { Asociación de las lesbianas con el } \\
\text { estereotipo de «machorras», es decir, } \\
\text { bruscas, con comportamientos de } \\
\text { hombres y con gusto por otras } \\
\text { mujeres. }\end{array}$ & $\begin{array}{l}\text { - No se presentaron referencias a la resignificación de las } \\
\text { lesbianas. }\end{array}$ \\
\hline
\end{tabular}

Tabla 2. Imaginarios de interculturalidad. 
llarse como audiencias interactivas. El hecho de que niños y niñas hicieran públicas sus opiniones a través de la televisión, les implicó el reto de ser coherentes (al menos frente a sí mismos, sus pares, padres y profesores) en torno a las relaciones que establecen con ella en su vida cotidiana, evidenciando en su consumo las críticas expresadas. Esto equivaldría a procesos de interactividad que comprometen el tejido social, más allá de la interacción tecnológica individual.

El proyecto tuvo limitaciones relacionadas con el tamaño reducido de la muestra, por lo que se recomienda ampliarla a otras poblaciones, de tal manera que se pueda profundizar en análisis comparativos, pues aunque se desarrolló una iniciativa similar en la ciudad de Montería (Colombia), no fue posible establecer comparaciones en todas las categorías. Igualmente, se reconoce como limitación el que no se haya podido efectuar un cabal seguimiento de las respuestas de otros públicos (productores televisivos, padres, madres y adultos en general) a las reflexiones expuestas por niños y niñas en sus vídeos. Según plantean Ferber y otros (2007) es así como se garantizaría una interactividad en la que una tercera parte responda al mensaje hecho público por la interacción de otras dos. En aras de mejorar la estrategia, también se considera necesario involucrar de forma más profunda a los niños y niñas en la producción de los vídeos, de tal manera que integren auténticamente sus propias estéticas y sentidos. Se propone entonces, la continuidad de esta iniciativa a partir de la consolidación de compromisos y alianzas que permitan enfatizar debates ciudadanos que tengan como punto de partida las opiniones infantiles sobre la televisión.

\section{Notas}

${ }^{1}$ Este observatorio no se concibió como una estructura orgánica centralizada, sino como un ejercicio de investigación participativa destinado a validar una metodología de observatorio infantil de televisión. 2 La metodología del estudio imposibilita generalizaciones. Cuando se menciona a niños y niñas se hace referencia a los resultados co- munes para el conjunto de niños y niñas participantes en el proyecto, independientemente de sexo, escolaridad, estrato socioeconómico y tipo de institución educativa.

3 Telecaribe fue el primer canal regional de Colombia, el cual tiene experiencia en la realización de programas de carácter educativo. Una de sus producciones infantiles llamada "El libro de Sofía» ganó el premio India Catalina al mejor programa infantil colombiano en 2007. Teniendo en cuenta lo anterior se consideró prioritario que el canal se vinculara como aliado de "Observar TV".

${ }^{4}$ Se puede acceder a los vídeos en: www.youtube.com/channel/UC-yypSIIO0unQdALOY8GAPw.

5 Entre otros, «Los Simpson», «Power Rangers» y «Dragon Ball Z».

6 Términos utilizados para definir lo siguiente: rateros, como ladrones; gamines, como niños y niñas de la calle; guerrilleros y paramilitares, como dos de las fuerzas armadas del conflicto colombiano.

7 Expresiones usadas para referirse a lo siguiente: cachacos como habitantes del centro del país; costeños como habitantes de la costa caribe; y corronchos/as como personas cuyo comportamiento es percibido extravagante o burdo en relación con las normas sociales (adjetivo despectivo)

\section{Apoyos y agradecimientos}

Este proyecto fue realizado en el marco del programa de apoyo a jóvenes investigadores, convocatoria 2007, de la Dirección de Investigaciones y Proyectos de la Universidad del Norte (UN-OJ-2007. 04115), actuando como joven investigadora la comunicadora social Vanessa Castro Morales, bajo la dirección de Jair Vega y la asesoría de Andrea Lafaurie. Además, fue establecido un convenio con el canal regional de televisión Telecaribe (2008-07708) que permitió la producción y emisión de los vídeos de los niños y niñas del observatorio, participando también en la realización de los mismos el Colectivo Audiovisual Pimentón Rojo.

\section{Referencias}

Aguaded, J.I. (1999). Convivir con la televisión. Barcelona: Paidós. AgUADED, J.I. (2005). Enseñar a ver la televisión: una apuesta necesaria y posible. Comunicar, 25, 51-55.

AIKAT, D. (2005). "Click here for fun, games, friends...» Analyses of Media Content Characteristics of Children's Web Sites. International Communication Association, 2005 Annual Meeting.

Alfaro, R.M. E QuezadA, A. (2006). jAmpay, tele!, niños y adolescentes opinan y juzgan a la televisión peruana. Lima: Veeduría Ciudadana de la Comunicación Social y la Asociación de Comunicadores Sociales Calandria.

Bourdieu, P. (1984). A Social Critique of the Judgement of Taste. USA: Harvard College and Routledge $\mathcal{E}$ Kegan Paul Ltd.

BUCKINGHAM, D. (2005).

Educación en medios. Alfabetización, aprendizajey cultura contemporánea. Barcelona: Paidós.

Chang, A., CHI, P.Y., MONTFORT, N., BREAZEAL, C. \& LIEBERMAN, H. (2010). Lessons Learned from Designing Children's Interactive Narratives. The International Conference $\&$ Festival of the Electronic Literature Organization.

Charles M. \& Orozco,

Tabla 3. Imaginarios de participación: responsabilidad democrática. 
G. (1990). Educar para la recepción: hacia una lectura crítica de los medios. México: Trillas.

Dezuanni, M. E Monroy-HernándeZ, A. (2012). Prosumidores interculturales: creación de medios digitales globales entre jóvenes. Comunicar, 38, 59-66.

Ferber, P., Foltz, F. \& Pugliese, R. (2007). Cyberdemocracy and Online Politics: A New Model of Interactivity. Bulletin of Science, Technology \& Society, 27, 391-400.

FERRÉs, J. (1994). Televisión y educación. Barcelona: Paidós.

Koolstra, C. \& Bos, M. (2009). The Development of an Instrument to Determine Different Levels of Interactivity. The International Communication Gazette, 71(5), 373-391.

Livingstone, S., Bober, M. \& Helsper, E. (2005). Active Participation or Just More Information? Young People's Take-up of Opportunities to Act and Interact on the Internet. Information, Communication \& Society, 8, 287-314.

López-de-la-Roche, M., Martín-Barbero, J., Rueda, A. \& VALENCIA, S. (1998). Los niños como audiencias. Colombia: Ministerio de Comunicaciones / Universidad del Valle.

MASTERMAN, E. \& Rogers, Y. (2002). A Framework for Designing Interactive Multimedia to Scaffold Young Children's Understanding of Historical Chronology. Instructional Science, 30, 221-241.

Ministerio dE Educación NACIONAL (Ed.) (2004). Estándares básicos de competencias ciudadanas. Guía, 6. Formar para la ciudadanía... isi es posible! Lo que necesitamos saber y saber hacer. Colombia: MEN

Mouffe, C. (1999). Por una política de identidad democrática. Seminario "Globlalización y diferenciación cultural»; marzo. MACBA-CCCB, 1999. (www.macba.es/antagonismos/castellano/09 04.html) (05-06-2012).

MũÑZ, G. E MuÑoz, D. (2008). La ciudadanía juvenil como ciudadanía cultural: una aproximación teórica desde los estudios culturales. Revista Argentina de Sociología, 11, 217-236.
Orozco, G.; Navarro, E. E García-Matilla, A. (2012). Desafíos educativos en tiempos de auto-comunicación masiva: la interlocución de las audiencias. Comunicar, 38, 67-74.

PINEDA, N. (1999). Tres conceptos de ciudadanía para el desarrollo de México. (www.colson.edu.mx/Estudios\%20Pol/Npineda/Estepai2.pdf) (05-06-2012).

PINTOS, J.L. (2000). Construyendo realidad(es): los imaginarios sociales. Santiago de Compostela. (http://idd00qmm.eresmas.net/articulos/medios.htm) (05-06-2012).

PotTer, W. J. (1998). Media Literacy. London: Sage.

PRENSKY, M. (2001). Digital Natives, Digital Inmigrants. On the Horizont, $\mathrm{MCB}$ University Press, 5. (www.marcprensky.com/writing/prensky $\% 20-\% 20$ digital\%20natives, $\% 20$ digital $\% 20$ immigrants $\% 20$ \%20part1.pdf) (05-03-2011).

Riaño, P. (2000). Memorias metodológicas. Revista de Estudios Sociales, 7. Bogotá: Universidad de los Andes, 48-60.

Rincón, O. (2008). No más audiencias, todos devenimos productores. Comunicar, 30, 93-98.

RODRíGUEZ, A. (2005). Los efectos de la televisión en niños y adolescentes. Comunicar, 25.

VeGA, J. E García, L.H. (2008). ¿Qué tiene que ver Popeye con la ciudadanía? Alterfacto, 5, 21-28. Universidad Cooperativa de Colombia

VeGa, J. \& CASTro, V. (2006). Cuentos ciudadanos. Desarrollo de competencias ciudadanas y reconstrucción de imaginarios de ciudad y ciudadanía, a través del periodismo escolar. Revista Investigación y Desarrollo, 14(2), 330-351.

VEGA, J. \& MENDIVIL, C. (2012). jovenHABLAjoven: Lessons Learned about Interpellation, Peer Communication, and Second-generation Edutainment in Sexuality and Gender Projects among Young People. In S. WAISBORD, E R. OBREGón (Eds.), The Handbook of Global Health Communication. (pp. 444-468). USA: Wiley-Blackwell. 